\title{
Presentación
}

\section{Boris Graizbord*}

La ciudad es un escenario multifacético en el que concurren intereses diversos que dan lugar tanto a los diferentes procesos sociales como a representaciones simbólicas mediante las que se establecen e imponen valores de individuos o grupos de acuerdo con su mayor o menor poder económico, político y cultural. Al mismo tiempo, la ciudad genera valor, pues ofrece condiciones para convertir trabajo en mercancías, éstas en dinero y dinero en capital, lo que en gran medida determina las decisiones económicas, los comportamientos sociales y las formas de vida de sus habitantes. En efecto, en la ciudad se evidencia el esfuerzo cotidiano de los individuos, familias y grupos heterogéneos que, independientemente de sus atributos, buscan imponerse, reproducirse y sobrevivir.

En esta relación de reciprocidad -dialéctica, dirían algunos- entre las formas espaciales y los procesos sociales, el análisis urbano exige la convergencia pluridisciplinaria de diferentes enfoques cuantitativos y cualitativos pero complementarios y de acciones concurrentes del sector público, la iniciativa privada y la sociedad civil en general -como somos testigos cada vez que algún evento trastoca la vida cotidiana de los ciudadanos.

Los conceptos anteriores, válidos para todas las sociedades, sistemas políticos y grados de desarrollo económico, adquieren diferente significado en función del tamaño, especialización, base económica y estatus político-administrativo de la ciudad. Siendo así, no sólo tiene sentido, sino que es pertinente, la distinción entre las grandes metrópolis y las ciudades de tamaño intermedio. Estas últimas fueron objeto de análisis de un simposio sobre "Antropología urbana de las ciudades medias", que organizamos el que esto suscribe y la doctora Carmen Icazurriaga dentro del programa del XIII Congreso Internacional de Ciencias Antropológicas y Etnológicas (CICAE), celebrado en la ciudad de México la semana del 29 de julio al 5 de agosto de 1993.

En su discurso inaugural, la doctora Lourdes Arispe, presidenta de la Unión Internacional de Ciencias Antropológicas y Etnológicas, tocó varios temas de relevancia actual para los antropólogos y, en general, para los científicos sociales. Mencionó la necesidad de estudios que respondan a la creciente importancia de las ciudades medias tanto en los proyectos de desarrollo nacional como en una nueva configuración del espacio geográfico de los países en desarrollo. En efecto, estas ciudades parecen tomar un lugar protagónico en el análisis de los procesos de cambio que en la actualidad experimentan todos los países del mundo. Más aún, si se acepta que la "transición demográfica" y su secuela en términos de movilidad y distribución geográfica de la población afectan o

*Profesor-investigador del Centro de Estudios Demográficos y de Desarrollo Urbano de El Colegio de México. 
afectarán en un momento a los países en desarrollo, es indiscutible la importancia de estudiar a fondo -cuantitativa y cualitativamente- las ciudades intermedias o secundarias que son las que verán desafiada su histórica pasividad y estatus de acompañantes o testigos aparentemente silenciosos de los procesos de urbanización, industrialización y de desarrollo que caracterizan a las sociedades contemporáneas.

Para la organización del simposio se tomaron en consideración los siguientes aspectos. En primer lugar, era importante la representatividad geográfica; así se procuró que hubiese estudios referentes a ciudades de diversas regiones del país. En segundo lugar, era importante la presencia multidisciplinaria; con ese propósito, se invitó a antropólogos sociales así como a sociólogos, historiadores, urbanistas, etcétera. En tercer lugar, se consideró necesario presentar casos y puntos de vista no sólo nacionales sino de otros países; así, invitamos a reconocidos investigadores internacionales (cinco norteamericanos y un catalán), tres de los cuales en equipo presentaron resultados de su prolifica investigación sobre una región y una ciudad mexicanas.

Los trabajos que se incluyen en este número especial de Estudios Demográficos y Urbanos son producto de dicho simposio, y no obstante su diversidad, los hemos clasificado según los siguientes criterios: los que parten de un modelo conceptual sobre la ciudad media y presentan un estudio de caso y los que a partir del conocimiento empírico discuten o plantean un aspecto relevante de algún proceso urbano que pudiera alcanzar un carácter general y aportar elementos para formalizar eventualmente un modelo analítico. Por otra parte, se incluyen estudios de ciudades en varias regiones del país, lo que permite tener una visión de la complejidad de los problemas del conjunto de las ciudades medias de acuerdo con procesos regionales específicos. Hay algunos que abordan problemas actuales sin dejar de lado en su explicación el corte diacrónico, y otros más se refieren a diversos actores sociales.

Cabe enfatizar que los participantes en el simposio y los trabajos aquí presentados se distinguen por su origen y enfoque interdisciplinarios. Si bien esto, de entrada, no permite reconocer una homogeneidad metodológica, sí enriquece el panorama que enfrentamos los interesados en conocer y estudiar a las ciudades medias.

No es claro en dónde empieza y acaba el rango de las ciudades medias. El primer trabajo es un análisis general y valioso, por ser propositivo, que cuestiona el concepto y las ideas en torno a la clasificación de las ciudades por su tamaño. Plotnicov sugiere que es la ubicación regional de las ciudades, independiente de su tamaño, lo que determina su desarrollo. Así, destaca que las ciudades de la región centro-norte de México tendrían algunas ventajas competitivas para captar un segmento del mercado de consumo y del gasto disponible de un grupo de población norteamericano, de la tercera edad, que busca retirarse a áreas con buen clima y menores costos de vida. Si accedieran a recibirlos, los gobiernos locales de estas ciudades tendrían que orientar sus inversiones hacia la oferta de bienes y servicios para estos grupos, lo cual crearía empleos en el sector terciario, mayores ingresos fiscales y bienestar para la población local. 
White, por su parte, en un excelente trabajo, propone una vía metodológica para comprender y explicar las estructuras sociales de las comunidades urbanas, a partir del enfoque de redes de matrimonios y parentesco. Esta teoría se apoya en la interdependencia entre las actividades humanas y los vínculos sociales que se establecen sobre la base de las relaciones matrimoniales. La ciudad -como afirma White-constituye un escenario en el cual se generan corrientes de actividad humana dentro de las cuales los individuos se desempeñan. Este desempeño depende en gran medida de las relaciones familiares. El autor distingue entre éstas y las interacciones de otro tipo que, dice, son efímeras o bien no dejan huella ni exigen un compromiso más allá de la responsabilidad que dictan las reglas formales de convivencia cívica y del mercado. Propone el método de random walks que le ha permitido rastrear líneas genealógicas y de ascendencia en diferentes tipos de ocupación laboral en la ciudad.

Concluye que el enfoque de redes permite, por un lado, asimilar cuestiones muy personales de los individuos pero, al mismo tiempo, identificar o dar sentido a estrategias y posiciones de ciertos grupos para preservar, adquirir o acrecentar su influencia social y su poder económico y político.

En su trabajo, Sariego describe tres modelos de organización de la actividad minera que ha sido, desde la colonia, estructuradora de asentamientos urbanos más o menos estables en nuestro país. La mayoría, por supuesto, ha dejado su impronta en el territorio nacional, y si bien su importancia ha disminuido relativamente, su riqueza se refleja ahora en lo que constituye un patrimonio arquitectónico y cultural que es escenario de intentos contemporáneos de supervivencia en algunos casos, y de exitosos esfuerzos para refuncionalizar las economías urbanas en otros.

Feixa, por su parte, utilizando el ejemplo de una localidad catalana (Lleida), se pregunta sobre la construcción de identidades culturales en las ciudades medias y la participación de diversos grupos en la articulación de las mismas. Enfatiza la emergencia de los sectores juveniles como sujeto social que crea espacios en la escena urbana.

Murphy, Winter y Morris investigan las relaciones entre unidades domésticas así como su organización para asimilar los cambios que el sistema mundial imprime en la economía del sistema regional de los valles centrales del estado de Oaxaca. Encuentran, entre otros interesantes hallazgos, que la migración temporal y la movilidad espacial de la fuerza de trabajo representa la estrategia principal de supervivencia de la unidad familiar en tiempos de crisis, como la que afectó a nuestro país en los años ochenta.

Esta crisis, que tuvo efectos también en la agroindustria henequenera de Yucatán, modificó profundamente las estructuras económicas regionales, lo que a su vez, por lo menos hasta fines de los años setenta, repercutió en Mérida la ciudad capital y la más importante de la península de Yucatán. La gestión del empresariado industrial -nos dice Ramírez- ha sido el motor de los cambios que mejoraron las perspectivas económicas de la ciudad y la región. Con todo - pensamos- fue, además de la actividad petrolera en el sureste, la enorme inversión turística en Cancún, fo- 
mentada por el Estado, lo que coadyuvó indirectamente a estimular el crecimiento económico y el desarrollo de las actividades urbanas en esta histórica ciudad media.

Ruiz explora la forma en que la modernización económica ha tenido desiguales efectos sobre los hombres y las mujeres de la clase media de Hermosillo. En la primera parte de su trabajo se describen la ideología y la práctica de género que, previas a la modernización, segregaban jerárquicamente a las mujeres en espacios privados, si bien éstas participaban con los hombres en trabajos y labores comunes. La modernización agrícola transformó estas relaciones de género. La clase media se profesionalizó y convirtió en asalariada, de tal suerte que al convertir al jefe familiar en trabajador fuera del hogar se privilegió lo productivo no doméstico sobre lo reproductivo doméstico, afectando así la percepción y valoración del trabajo femenino dentro del hogar.

La segregación existe no sólo dentro de la familia sino también en el espacio de la ciudad. Alegría modela y explora empíricamente la relativa homogeneidad-heterogeneidad de los espacios residenciales en Tijuana. Argumenta, desde una perspectiva ecológico-demográfica, que la diferenciación-estructuración del espacio urbano resulta de una práctica social de los residentes que se disputan los recursos urbanos, en el contexto tanto del mercado como de los mecanismos públicos institucionales. Al reconocer la inmovilidad de tales recursos urbanos, la disputa por ellos incorpora la dimensión espacial en términos de cercanía-lejanía de los residentes urbanos a la presencia de aquéllos. De esta suerte, los que tienen mayores ingresos y (o) conocimiento buscarán mejorar su posición relativa (accesibilidad) dentro del espacio de la ciudad, dando lugar a una estructura urbana que refleja estos procesos.

El "estilo de vida" se relaciona con la urbanización y la forma urbana. Quintal explora esta relación en el caso de Poza Rica, ciudad petrolera mexicana. Su estudio destaca el papel que ha tenido en la estructuración de la ciudad la presencia y fuerza política de un grupo social "clave": el sindicato de los trabajadores petroleros, que ha dominado y acaparado los puestos "clave" de los gobiernos de la ciudad.

Finalmente, Icazuriaga describe, por un lado, el desarrollo urbano de la ciudad de Querétaro, destacando el crecimiento y la diversificación de su economía, que ha favorecido al crecimiento y a la concentración de los sectores medios. Por otro lado, analiza, a partir de una muestra aplicada en 1992, los cambios en el estilo de vida de estos sectores. La conclusión implícita de este trabajo apunta a la relación positiva entre crecimiento económico urbano y ampliación de las clases medias, la cual induce una mejoría en la distribución del ingreso entre los habitantes de la ciudad. Sin embargo, la posibilidad de generalizar las conclusiones anteriores estaría supeditada a la forma en que las ciudades medias se vinculan al crecimiento económico general y, quizá, al desarrollo de relaciones funcionales con la ciudad primaria, pero también con sus regiones tributarias.

En resumen, los trabajos anteriores representan sólo una pequeña muestra de la amplitud temática y las múltiples vías de abordaje posibles y necesarias para alcanzar un conocimiento cabal de los procesos urba- 
nos y del potencial que estas ciudades pueden tener en el desarrollo futuro de nuestras sociedades. Su recopilación intenta cumplir con el deber de difundir conocimiento y, no menos, con el reconocimiento del valor que los organizadores del XIII CICAE tuvieron de no hacer distingos disciplinarios al invitarnos. 
